\title{
ACCESSIBILITY AND FOLIATIONS WITH SINGULARITIES
}

\author{
BY P. STEFAN \\ Communicated by Alberto Calderon, March 13, 1974
}

Introduction. Recently, Sussmann has proved that the accessible sets of a system of vectorfields on a $C^{\infty}$ manifold $M$ are immersed submanifolds of $M$ [1], [2]. In this paper we state a general theorem on accessible sets of collections of 'arrows' and indicate how it implies (a) the above result; (b) the fact that the orbits of an arbitrary isotopically connected' subgroup of $\operatorname{Diff}(M)$ form a foliation with singularities; and (c) a similar result for groupoids of germs of local diffeomorphisms. The complete proofs will be published elsewhere.

The results of this paper were obtained independently of Sussmann's work.

1. Statement of the main result. Throughout this paper, the word 'differentiable' refers to a fixed class $C^{q}, 1 \leqq q \leqq \infty$, and $M$ is a finitedimensional paracompact differentiable manifold. Theorems 1 and 4 are also valid in the real analytic case.

A subset $L$ of $M$ is said to be a $k$-leaf of $M$ if there exists a differentiable structure $\sigma$ on $L$ such that (i) $(L, \sigma)$ is a connected $k$-dimensional immersed submanifold of $M$, and (ii) if $N$ is an arbitrary locally connected topological space, and $f: N \rightarrow M$ is a continuous function such that $f(N) \subset L$, then $f: N \rightarrow(L, \sigma)$ is continuous.

It follows from the properties of immersions that if $f: N \rightarrow M$ is a differentiable mapping of manifolds such that $f(N) \subset L$, then $f: N \rightarrow(L, \sigma)$ is also differentiable. In particular, $\sigma$ is the unique differentiable structure on $L$ which makes $L$ into an immersed $k$-dimensional submanifold of $M$. Since $M$ is paracompact, every connected immersed submanifold of $M$ is separable, and so the dimension $k$ of a leaf $L$ is uniquely determined.

We say that $\boldsymbol{F}$ is a $C^{q}$-foliation of $M$ with singularities if $\boldsymbol{F}$ is a partition of $M$ into $C^{q}$-leaves of $M$ such that, for every $x \in M$, there exists a local $C^{q}$-chart $\psi$ of $M$ with the following properties:

(a) The domain of $\psi$ is of the form $U \times W$, where $U$ is an open neighbourhood of 0 in $R^{k}, W$ is an open neighbourhood of 0 in $R^{n-k}$, and $k$ is the dimension of the leaf through $x$.

AMS (MOS) subject classifications (1970). Primary 57D30, 58A30, 49E15. 
(b) $\psi(0,0)=x$.

(c) If $L$ is a leaf of $\boldsymbol{F}$, then $L \cap \psi(U \times W)=\psi(U \times l)$, where $l=\{w \in W$ : $\psi(0, w) \in L\}$.

We write $(M, F)$ for the $C^{a}$-manifold with the same underlying set as $M$ and with the $C^{q}$-structure of the disjoint sum of leaves of $\boldsymbol{F}$.

By a local diffeomorphism of $M$ we mean a diffeomorphism of one open subset of $M$ onto another. We say that a differentiable function $a: R \times$ $M \rightarrow M$ is an arrow if its domain is an open subset of $R \times M$ and if it satisfies the following two conditions: (i) for every $t \in R, a^{t}=a(t,-)$ is a local diffeomorphism of $M$ (possibly with the empty domain), and (ii) if $(t, x)$ belongs to the domain of $a$, then so does $(s, x)$ for every $s$ between 0 and $t$ and $a(0, x)=x$. We write $\dot{a}(t, x)$ for the tangent vector at $t$ of the curve $a(-, x)$, and $\left(a^{t}\right)^{*}(x)$ for the differential at $x$ of the function $a^{t}: M \rightarrow M$. If $y=a(t, x)$ then $\dot{a}(t, x) \in T_{y} M$, and $\left(a^{t}\right)^{*}(x)$ is a linear mapping $T_{x} M \rightarrow$ $T_{y} M$.

If $A$ is a set of arrows on $M$, we let $\Psi A$ denote the collection of all the local diffeomorphisms $\phi$ of the form

$$
\phi=a_{1}^{t_{1}} \circ a_{2}^{t_{2}} \circ \cdots \circ a_{p}^{t_{p}}
$$

for some $a_{i} \in A$ and $t_{i} \in R . A(x)$ and $\bar{A}(x)$ denote the vector subspaces of $T_{x} M$ spanned respectively by the set $\{\dot{a}(t, y): a \in A, a(t, y)=x\}$ and by $\bigcup \phi^{*}(y) \cdot A(y)$, where the union extends over all $\phi$ and $y$ such that $\phi \in \Psi A$ and $\phi(y)=x$.

We say that $A$ is symmetric if $\phi \in \Psi A$ implies $\phi^{-1} \in \Psi^{\circ} A$ and that it is homogeneous if $\left(a^{t}\right)^{*}(x) \cdot A(x) \subset A(y)$ whenever $a \in A$ and $a(t, x)=y$.

We write $y=x \bmod A$ if $y=x$ or if $y=\phi(x)$ for some $\phi \in \Psi A$. If $A$ is symmetric, then this is an equivalence relation on $M$; its equivalence classes are termed here the accessible sets of $A$.

Let $\sim$ be an equivalence relation on $M$. We say that an arrow $a$ (or a local diffeomorphism $\phi$ ) respects $\sim$ if $a(t, x) \sim x$ whenever $(t, x)$ belongs to the domain of $a$ (or $\phi(x) \sim \phi(y)$ whenever $x \sim y$ and both $x$ and $y$ belong to the domain of $\phi$ ).

Proposition. Let $\sim$ be an equivalence relation on $M$ and let $\tilde{A}$ be the class of all arrows on $M$ which respect $\sim$. Then $\tilde{A}$ is symmetric and homogeneous.

COROLlaRY. Every symmetric set of arrows is contained in a homogeneous symmetric set of arrows whose accessible sets are the same.

Proof of the Corollary. Take $\sim$ to be the relation $x=y(\bmod A)$.

THEOREM 1. (a) Let $A$ be a symmetric set of arrows on $M$ and let $\boldsymbol{F}=\boldsymbol{F}(A)$ be the partition of $M$ into the accessible sets of $A$. Then $\boldsymbol{F}$ is a 
foliation with singularities. In particular, every accessible set of $A$ is a leaf of $M$, and thus admits a unique differentiable structure of a connected immersed submanifold of $M$.

(b) $T_{x}(M, F)=\bar{A}(x)$ for every $x \in M$. In particular, $T_{x}(M, F)=A(x)$ for every $x \in M$ if and only if $A$ is a homogeneous set of arrows.

(c) Let $\sim$ be an equivalence relation on $M$ and let $A=\tilde{A}$. If $\phi \in$ Loc $\operatorname{Diff}(M)$ and $\phi$ respects $\sim$, then $\phi \in \operatorname{Loc} \operatorname{Diff}(M, F)$.

2. Foliations generated by subgroups of $\operatorname{Diff}(M)$. Let $G$ be a subgroup of $\operatorname{Diff}(M)$. Two elements $g$ and $h$ of $G$ are said to be $G$-isotopic if there exists a differentiable mapping $a: R \times M \rightarrow M$ such that $a^{t} \in G$ for every $t \in R, a^{t}=g$ for $t \leqq 0$, and $a^{t}=h$ for $t \geqq 1$. The isotopy component $G_{0}$ of the identity is a normal subgroup of $G$.

THEOREM 2. (a) Let $\boldsymbol{F}=\boldsymbol{F}\left(G_{0}\right)$ be the partition of $M$ into $G_{0}$-orbits. Then $\boldsymbol{F}$ is a foliation with singularities.

(b) $G \subset \operatorname{Diff}(M, F)$ and every $G$-orbit consists of $G_{0^{-}}$orbits of the same dimension.

(c) If $G / G_{0}$ is countable, then every $G$-orbit admits a unique structure of a separable immersed submanifold of $M$.

Proof. Let $A$ be the set of all differentiable mappings $R \times M \rightarrow M$ such that $a^{t} \in G$ for every $t \in R$, and $a^{t}=\mathrm{id}_{M}$ for $t \leqq 0$. Then $A$ is a symmetric set of arrows and the accessible sets of $A$ are the orbits of $G_{0}$.

3. Foliations generated by groupoids. If $\phi \in \operatorname{Loc} \operatorname{Diff}(M)$ and $x$ belongs to the domain of $\phi$, let $\gamma(x, \phi)$ denote the germ of $\phi$ at $x$. Let $e(x)=\gamma\left(x, \mathrm{id}_{M}\right)$, and let $\Delta=\Delta(M)$ be the groupoid of all germs of local diffeomorphisms of $M$. Let $\alpha: \Delta \rightarrow M$ be the projection onto the initial point, so that $\alpha(\gamma(x, \phi))=x$.

Let $\Gamma$ be a subgroupoid of $\Delta$. We say that $g \in \Gamma$ and $h \in \Gamma$ are $\Gamma$-isotopic if $\alpha(g)=\alpha(h)$ and if there exists an open neighborhood $U$ of $\alpha(g)$ and a differentiable mapping $a: R \times U \rightarrow M$ such that (i) $\gamma\left(\alpha(g), a^{t}\right) \in \Gamma$ for every $t \in R$, (ii) $\gamma\left(\alpha(g), a^{t}\right)=g$ for $t \leqq 0$, and (iii) $\gamma\left(\alpha(g), a^{t}\right)=h$ for $t \geqq 1$. Let $\Gamma_{0}=\{g \in \Gamma: g$ is $\Gamma$-isotopic to $e(\alpha(g))\}$. Then $\Gamma_{0}$ is a subgroupoid of $\Gamma$ and $g$ and $h$ are $\Gamma$-isotopic if and only if $\Gamma_{0} g=\Gamma_{0} h$.

THEOREM 3. The assertions of Theorem 2 remain valid if we replace $G$ by $\Gamma, G_{0}$ by $\Gamma_{0}$ and $\operatorname{Diff}(M, F)$ by $\Delta(M, F)$.

4. Foliations generated by systems of vectorfields. If $X$ is a differentiable vectorfield on $M, \exp X$ denotes the flow of $X$, so that $t \rightarrow \exp X .(t, x)$ is the integral curve of $X$ passing through $x$ at $t=0$. If $S$ is a set of vectorfields on $M$, we write $S(x)$ for the subspace of $T_{x} M$ spanned by $\{X(x)$ : $X \in S\}$, and put $\exp S=\{\exp X: X \in S\}$. It is clear that $\exp S$ is a symmetric 
set of arrows; the accessible sets of $S$ are, by definition, the accessible sets of $\exp S$.

THEOREM 4. (a) Let $\boldsymbol{F}=\boldsymbol{F}(S)$ be a partition of $M$ into the accessible sets of $S$. Then $\boldsymbol{F}$ is a foliation with singularities.

(b) $T_{x}(M, F)=S(x)$ for every $x \in M$ if and only if $\left(\exp X^{t}\right)^{*}(x) . S(x) \subset$ $S(y)$ whenever $X \in S$ and $\exp X .(t, x)=y$.

COROLlary. Let $\sim$ be an arbitrary equivalence relation on $M$, and let $S$ be the set of all vectorfields on $M$ that leave the equivalence classes of $\sim$ invariant. Then $S$ is closed under formation of the Lie bracket.

\section{REFERENCES}

1. H. J. Sussmann, Orbits of families of vector fields and integrability of systems with singularities, Bull. Amer. Math. Soc. 79 (1973), 197-199. MR 46 \#10020.

2. - Orbits of families of vector fields and integrability of distributions, Trans. Amer. Math. Soc. 180 (1973), 171-188.

School of Mathematics and Computer Science, University College of North Wales, Bangor LL57 2UW, United Kingdom 